\title{
Compact Bandpass Filters Based on Dual-Plane Microstrip/Coplanar-Waveguide Structure With Quarter-Wavelength Resonators
}

\author{
Tsung-Nan Kuo, Shih-Cheng Lin, Chi-Hsueh Wang, and Chun Hsiung Chen, Fellow, IEEE
}

\begin{abstract}
Compact wideband bandpass filters are proposed based on the dual-metal-plane structure consisting of both microstrip and coplanar-waveguide (CPW) quarter-wavelength resonators. By combining the advantages of dual-plane microstrip/CPW structure and stepped-impedance resonators, strong couplings between resonators may be accomplished so that the fourth-order cross-coupled filter with compact size and wide bandwidth may be realized. To further improve the selectivity, the sixth-order cross-coupled filter composed of four microstrip and two CPW resonators is implemented. Specifically, the implemented filters have the merits of compact size, good insertion/return losses, wide fractional bandwidth, and better selectivity. Good agreement between simulated and measured responses of these filters is demonstrated.
\end{abstract}

Index Terms-Bandpass filter (BPF), coplanar waveguide (CPW), cross coupling, microstrip, quarter-wavelength steppedimpedance resonator.

\section{INTRODUCTION}

$\mathbf{I}$ $\mathrm{N}$ modern wireless communication systems, bandpass filters (BPFs) with miniaturized size and good performance are required to reduce the fabrication cost. Various microstrip filter structures based on half-wavelength $(\lambda / 2)$ resonators have been proposed, using the parallel-coupled [1] and open-loop resonator [2] topologies. However, these filters have the drawback of large circuit size.

Comparing with $\lambda / 2$ resonator filter, the filters consisting of quarter-wavelength $(\lambda / 4)$ resonators have the merit of more compact size. In [3], microstrip stepped-impedance resonators were adopted to reduce the filter size. In [4], the microstrip net-type resonators were employed in the filter design. Although the above microstrip filters with $\lambda / 4$ resonators have the advantage of compact size, they are implemented based on the narrow band approximation.

Coplanar-waveguide (CPW) is another popular guide structure for filter design. Compared with the microstrip structure, CPW possesses the merits of insensitivity to the substrate thickness, easy construction of short-circuited elements, and etc. In

Manuscript received November 3, 2006; revised November 18, 2006. This work was supported by the National Science Council of Taiwan, R.O.C., under Grants NSC 95-2752-E-002-001-PAE, NSC 95-2219-E-002-008, and NSC 95-2221-E-002-196.

The authors are with the Department of Electrical Engineering and Graduate Institute of Communication Engineering, National Taiwan University, Taipei 106, Taiwan, R.O.C. (e-mail: chchen@ew.ee.ntu.edu.tw; r92942054@ntu.edu. tw).

Digital Object Identifier 10.1109/LMWC.2006.890460
[5], $\lambda / 2 \mathrm{CPW}$ resonators were adopted in the design of end-coupled filter. For the purpose of reducing the circuit size, the $\lambda / 4$ CPW stepped-impedance resonators were used in the filter implementation [6]. However, these CPW filters are also based on the narrow bandwidth design.

Recently, dual-plane structures are adopted in the filter design. In [7], the ultra-wideband filter with the multiple-mode resonator was reported using the microstrip-to-CPW transitions as inverter circuits. In [8], the broadside-coupled BPFs based on the $\lambda / 2$ microstrip and $\lambda / 4 \mathrm{CPW}$ resonators were proposed to increase the bandwidth. However, their circuit sizes are still large.

In this letter, compact wideband BPFs based on the dualmetal-plane structure consisting of both microstrip and CPW $\lambda / 4$ resonators are fabricated. By using the dual-plane layout with $\lambda / 4$ resonators, tight couplings between resonators may be realized so that the compact filter with wide bandwidth can be achieved. Moreover, by introducing the cross-coupled path and suitably arranging the physical distance between the resonators, the fourth-order filter with two transmission zeros is realized, having a fractional bandwidth of $30.06 \%$. To further improve the selectivity, the sixth-order filter with a fractional bandwidth of $34.6 \%$ is also implemented.

\section{Fourth-ORDER CROSS-COUPLED FILTER}

The structure of the proposed fourth-order cross-coupled dual-plane filter is shown in Fig. 1. For size reduction, the $\lambda / 4$ stepped-impedance resonators realized in both microstrip and CPW structures are adopted in the filter design. The microstrip resonators 1 and 4 as well as the CPW resonators 2 and 3 are all symmetric. The proposed filter is designed with a center frequency $f_{0}$ at $2 \mathrm{GHz}$ and a fractional bandwidth (FBW) of $28 \%$.

According to the specifications on $f_{0}$ and FBW, the proposed filter can be implemented using the coupled-resonator filter design procedures based on coupling coefficients $M_{i j}$ and the external quality factor $Q_{e}$ [9]. The design parameters associated with the specifications may be expressed as

$$
\begin{aligned}
Q_{e i} & =Q_{e o}=\frac{g_{0} g_{1}}{\mathrm{FBW}}=3.4276 \\
M_{12} & =M_{34}=\frac{\mathrm{FBW}}{\sqrt{g_{1} g_{2}}}=0.2397 \\
M_{14} & =\frac{\mathrm{FBW} \cdot J_{1}}{g_{1}}=-0.0615 \\
M_{23} & =\frac{\mathrm{FBW} \cdot J_{2}}{g_{2}}=0.22
\end{aligned}
$$




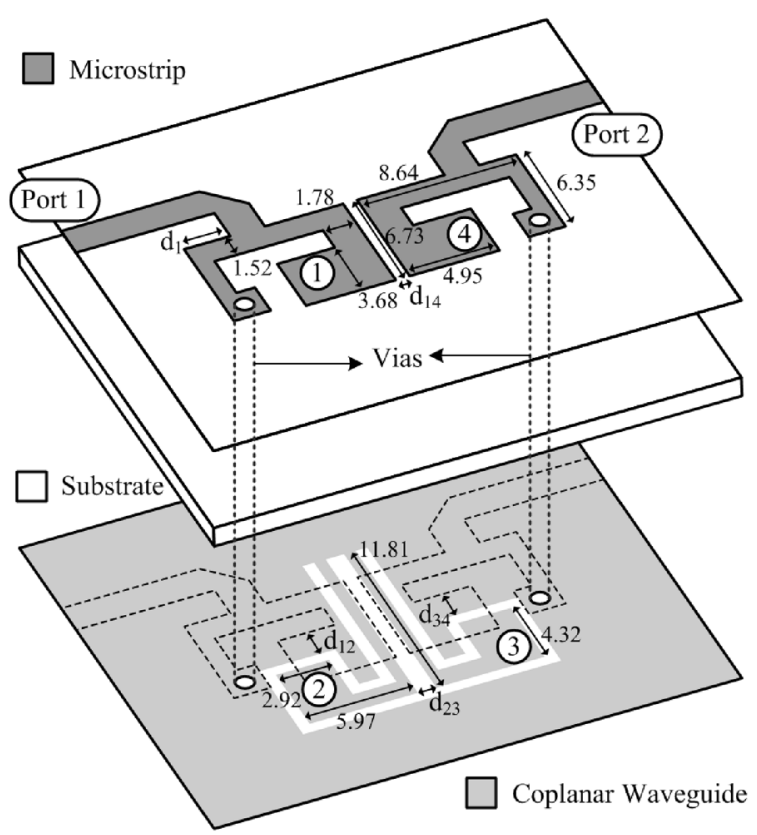

Fig. 1. Three-dimensional physical layout of the proposed fourth-order cross-coupled dual-plane filter. (Via diameter $=1, d_{1}=2.5, d_{12}=d_{34}=$ $1.78, d_{23}=1$, units: $\mathrm{mm}$.)

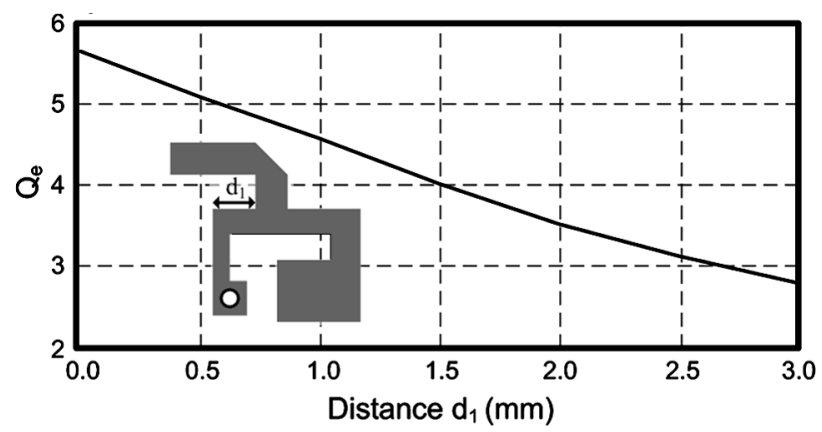

(a)

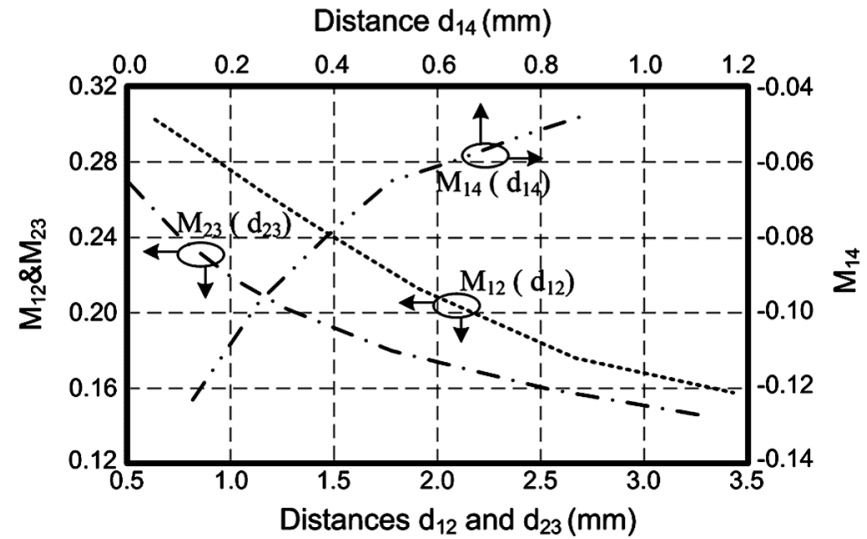

(b)

Fig. 2. Design curves for (a) external quality factor $Q_{e}$ and (b) coupling coefficients $M_{i j}$.

where $g_{i}$ and $J_{i}$ are the element values of the lowpass prototype filter [9]. In this study, the fullwave simulator ADS Momentum is used to extract the above parameters, from which one may obtain the design curves for the external quality factor $Q_{e}$ and coupling coefficients $M_{i j}$ as in Fig. 2.

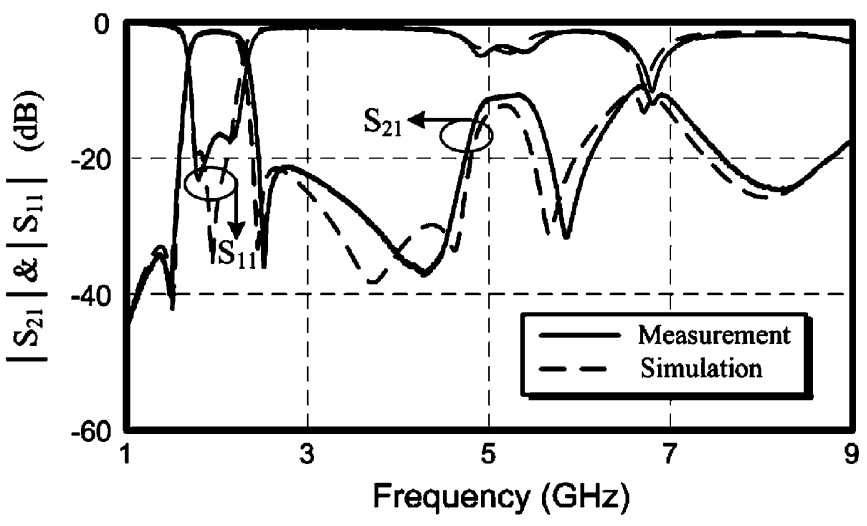

Fig. 3. Measured and simulated responses of the proposed fourth-order filter in Fig. 1.

It should be noted that tight couplings between resonators are needed to widen the filter bandwidth. Hence, to achieve the tight couplings, the magnetic coupling is enhanced by putting the short-circuited sections of folded CPW stepped-impedance resonators 2 and 3 as close as possible. The electric coupling is increased by minimizing the distance between the low-impedance sections of folded microstrip stepped-impedance resonators 1 and 4 . The mixed coupling is enhanced by increasing the overlapping area between the microstrip and CPW resonators.

Fig. 3 shows the measured and fullwave simulated responses of the fourth-order cross-coupled filter (see Fig. 1), which is fabricated on the FR4 substrate with $\varepsilon_{r}=4.4, \tan \delta=0.022$, and thickness $h=1 \mathrm{~mm}$. The measured center frequency is at $2.012 \mathrm{GHz}$ and the measured 3-dB fractional bandwidth is $30.06 \%$. The implemented filter has an insertion loss better than $1.42 \mathrm{~dB}$, return loss greater than $16.35 \mathrm{~dB}$ within the passband. The wideband fourth-order cross-coupled filter has a compact dimension of $18.288 \mathrm{~mm} \times 12.446 \mathrm{~mm}$, which is approximately $0.2245 \lambda_{g} \times 0.1528 \lambda_{g}$, where $\lambda_{g}$ is the microstrip guided wavelength on the substrate at center frequency.

Note that the first spurious passband associated with the proposed filter (Fig. 1) is observed unexpectedly around $5 \mathrm{GHz}$ due to the excitation of resonant coupled slotline mode along the slots of $\lambda / 4 \mathrm{CPW}$ resonators 2 and 3 . Theoretically, the filter composed of $\lambda / 4$ stepped-impedance resonators would push the first spurious passband beyond $3 f_{0}(=6 \mathrm{GHz})$. It is this unwanted coupled-slotline-mode resonance that degrades the stopband performance.

\section{SiXTh-ORder CROSS-COUPLED FILTER}

For further improving the selectivity, a sixth-order cross-coupled wideband filter is proposed. Fig. 4 show the physical layout of the proposed sixth-order filter. In this sixth-order filter design, $\lambda / 4$ short-circuited stepped-impedance resonators are adopted and folded to reduce the filter size. The resonators 1 and 6 and the resonators 2 and 5 are symmetric and implemented by the microstrip structure. The resonators 2 and 3 are also symmetric and realized by the CPW structure.

Like the design procedure for the fourth-order filter, the coupling coefficients and external quality factors can be calculated and extracted from the fullwave simulator. On implementing the sixth-order filter, the microstrip resonators 1 and 2 and the 


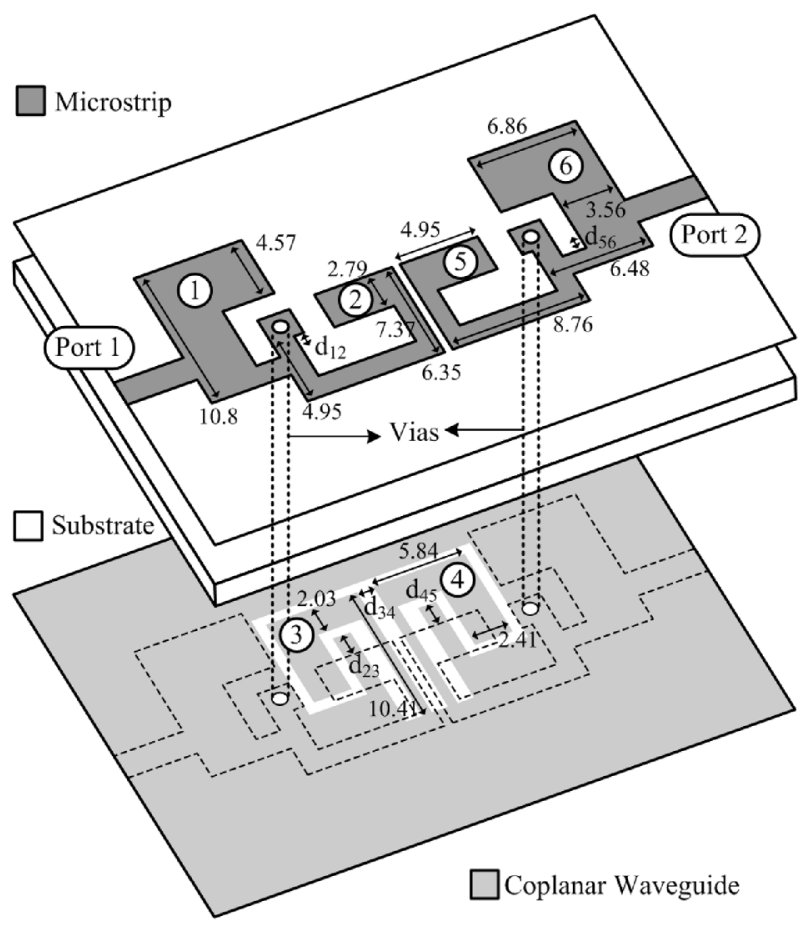

Fig. 4. Three-dimensional physical layout of the proposed sixth-order crosscoupled dual-plane filter. (Via diameter $=1, d_{12}=d_{56}=1.14, d_{23}=d_{45}=$ $1.52, d_{34}=1$, units: $\mathrm{mm}$.)

resonators 5 and 6 are connected to the joint short-circuited stub to give a large magnetic coupling. The CPW resonators 3 and 4 are used to achieve a large magnetic coupling by putting their short-circuited sections close to each other. The electric coupling is enhanced by putting the low-impedance sections of folded microstrip stepped-impedance resonators 2 and 5 as close as possible, and the mixed coupling is enlarged by increasing the overlapping area between the microstrip and CPW resonators.

The measured and fullwave simulated responses of the sixthorder cross-coupled dual-plane filter (see Fig. 4), also fabricated on the FR4 substrate, are shown in Fig. 5. The measured center frequency is at $1.983 \mathrm{GHz}$ and the measured $3-\mathrm{dB}$ fractional bandwidth is $34.6 \%$. The implemented filter has an insertion loss better than $1.78 \mathrm{~dB}$, return loss greater than $19.38 \mathrm{~dB}$ within the passband. The sixth-order filter has a compact dimension of $28.194 \mathrm{~mm} \times 13.081 \mathrm{~mm}$, which is approximately $0.346 \lambda_{g} \times$ $0.16 \lambda_{g}$.

\section{CONCLUSION}

In this letter, the compact wideband filters based on the dualmetal-plane structure consisting of both microstrip and CPW $\lambda / 4$ stepped-impedance resonators have been implemented and

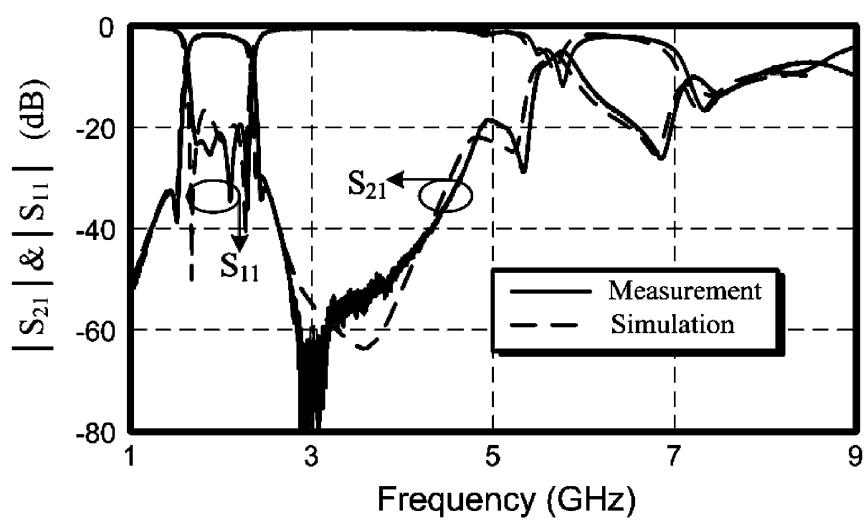

Fig. 5. Measured and simulated responses of the proposed sixth-order filter in Fig. 4.

carefully examined. With the dual-plane layout and by suitably designing the microstrip and CPW resonators, tight couplings between resonators may be achieved so that wide bandwidth filters with compact sizes may be realized. In addition, by an extension of the fourth-order filter, a sixth-order cross-coupled filter with large bandwidth, compact size, and good selectivity has been implemented. These proposed filters are useful for applications in communication system designs when wide bandwidth and good selectivity are required.

\section{REFERENCES}

[1] S. B. Cohn, "Parallel-coupled transmission-line-resonator filters," IEEE Trans. Microw. Theory Tech., vol. MTT-6, no. 4, pp. 223-231, Apr. 1958.

[2] J. S. Hong and M. J. Lancaster, "Couplings of microstrip square open-loop resonators for cross-coupled planar microwave filters," IEEE Trans. Microw. Theory Tech., vol. 44, no. 12, pp. 2099-2109, Dec. 1996

[3] C. F. Chen, T. Y. Huang, and R. B. Wu, "Compact microstrip crosscoupled bandpass filters using miniaturized stepped impedance resonators," in Proc. Asia-Pacific Microw. Conf., Nov. 2005, pp. 493-496.

[4] C. F. Chen, T. Y. Huang, and R. B. Wu, "Novel compact net-type resonators and their applications to microstrip bandpass filters," IEEE Trans. Microw. Theory Tech., vol. 54, no. 2, pp. 755-762, Feb. 2006.

[5] D. F. Williams and S. E. Schwarz, "Design and performance of coplanar waveguide bandpass filters," IEEE Trans. Microw. Theory Tech., vol. MTT-31, no. 7, pp. 558-566, Jul. 1983.

[6] J. Zhou, M. J. Lancaster, and F. Huang, "Coplanar quarter-wavelength quasi-elliptic filters without bond-wire bridges," IEEE Trans. Microw. Theory Tech., vol. 52, no. 4, pp. 1150-1156, Apr. 2004.

[7] H. Wang, L. Zhu, and W. Menzel, "Ultra-wideband bandpass filters with hybrid microstrip/CPW structure," IEEE Microw. Wireless Compon. Lett., vol. 15, no. 12, pp. 844-846, Dec. 2005.

[8] P. H. Deng, C. H. Wang, and C. H. Chen, "Novel broadside-coupled bandpass filters using both microstrip and coplanar-waveguide resonators," IEEE Trans. Microw. Theory Tech., vol. 54, no. 10, pp. 3746-3750, Oct. 2006.

[9] J. S. Hong and M. J. Lancaster, Microstrip Bandpass Filters for RF/Microwave Applications. New York: Wiley, 2001, ch. 8 and 10. 